the microelectronics industry has demonstrated, physical techniques can be used to provide the framework for this organization through the fabrication and machining of silicon materials. Arranging atoms through the use of nanomanipulative technologies is, in essence, just an extension of the manipulation. This approach to the production of a framework is closely linked to the ability to observe both the manipulations and the resulting structures, and there is clearly a reciprocity between the two.

Organizing frameworks could, however, also be self-assembling structures. ${ }^{8}$ This is familiar territory to biologists; phospholipid bilayer membranes are, perhaps, the best example, but some proteins and nucleic acids, too, can be thought of as self-assembling entities. And if vaccines can be thought of as machines for stimulating the immune system, then biologists have already developed self-assembling protein/nucleic acid nanodevices that are at least somewhat artificial.

Researchers can harness the understanding of how these molecules organize themselves to develop frameworks to their own designs. A group at the Scripps Research Institute (La Jolla, CA), ${ }^{5}$ for instance, used solid phase peptide synthesis to produce cyclic peptides containing L-glutamine and Lglutamic acid alternating with $\mathrm{D}$-alanines in an octameric ring. Controlled acidification of alkaline peptide solutions led to the spontaneous self-assembly of these ring peptides into tubes with an internal diameter of 0.7-0.8 nanometers. Individual ring subunits were held together by extensive hydrogen bonding and stacked in an antiparallel fashion. The authors suggested that tubes with a range of internal diameters and surface characteristics could be designed to mimic biological channels and pores, to control the growth of inorganic and metallic clusters, or to design optical and electronic devices. The accompanying box (see "Protein Crystals and Bioelectronic Arrays") shows how other researchers have attempted to use DNA for similar structural purposes.

\title{
Protein Crystals and Bioelectronic Arrays
}

Nanotechnology requires organizing principles, frameworks to provide K. Eric Drexler's (one of the founders of nanotechnology) "positional control" over atomic and molecular arrangements. Most current nanotechnology uses a "top-down approach," refining modern lithographic and machining techniques to make finer divisions. The alternative "bottomup approach" is exemplified by self-assembled molecules like those designed by Nadrian Seeman at New York University (New York), who has trained DNA to form a range of geometric shapes. DNA was chosen for a number of reasons, says Seeman: "the predictability of the intermolecular reactions, the ability to synthesize with ease desired sequences, and perhaps most importantly, the ability to use 'off-the-shelf' reagents to make unusual bases."

One of the designs he has synthesized is a polyhedron shaped like a structure seen in zeolite silicates. The truncated octahedron was synthesized using a solid support-based methodology. ${ }^{1}$ The squares were added in intermolecular addition reactions, and the hexagons were formed by seven successive intramolecular closure reactions. The synthesis involves a variety of molecular biology techniques, including hybridization, ligation, and the use of restriction enzymes.

The polyhedron has a large enough internal volume to act as a host for macromolecular guests. Molecules attached to the outside of the "cage" could be used to direct the complex to a target location. Another longer-term option would be to construct artificial multienzymes or multiprotein complexes by attaching fusion proteins to different edges of the polyhedron.

If the polyhedron can be catenated, forming a sort of molecular chain mail, one relatively near-term (next 3 to 5 years) application would be in facilitating the analysis of proteins that are hard to crystallize, including membrane proteins. The idea, Seeman explains, would be to produce fusions of the required protein with site-specific DNAbinding proteins. The DNAbinding elements would orientate the target protein within the lattice. " Those binding molecules that combine greater site specificity with the least structural perturbation would be optimal," says Seeman. He believes that this would make crystallization "an incrementally correctable experiment," rather than something that either works or does not. The development of such arrays might also pave the way for the construction of nanoscale memory devices in which the components are similarly bound to the framework. ${ }^{3}$

The immediate use of Seeman's DNA polyhedron structure is hampered by its lack of rigidity. As a result, the angles in the structure are sometimes indeterminate and the DNA tends to form inappropriate cyclic trimers or pentamers that disrupt growth of the lattice. Having tried to overcome this by protecting the DNA sticky ends, and having found that this approach was unfeasible in a large arrays, Seeman is now looking for more rigid molecules to use as components before he attempts to scale up the synthesis.

\section{-Musa Mhlanga}

1. Seeman, N.C. and Y. Zhang. 1994. Construction of a DNA truncated octahedron. J. Am. Chem. Soc. 116: 1661-1669.

2. Seeman, N.C. 1993.

Nanoengineering with DNA Biomolecular materials. In: Materials Research Society Symposium Proceedings; Material Research Society, Pittsburgh, PA. eds. M. Alper, H. Bayley, D Kaplan, M. Navia; p. 123-134.

3. Robinson, B.H. and N.C. Seeman. 1987. The design of a biochip: A self-assembling molecular-scale memory device. Protein Engineering 1:295-300.
Only the

imagination limits

the potential

applications of

bionanotechnology.

And, at present,

except for

biosensors, it is

only in the

imagination that

most of them

perform. 